\title{
Ética en Instituciones de Educación Superior para la Construcción de Relaciones de Confianza con Grupos de Interés (Stakeholders)
}

\author{
Natalia Marulanda ${ }^{(1)^{\star}}$ y Miguel D. Rojas ${ }^{(2)}$ \\ (1) Facultad de Ciencias Económicas y Administrativas, Corporación Universitaria Minuto de Dios, Carrera 45 No $22 \mathrm{D}$ - \\ 25 Bello, Colombia. (E-mail: nmarulandag@uniminuto.edu.co) \\ (2) Facultad de Minas, Dpto. de Ingeniería de la Organización, Univ. Nacional de Colombia, Carrera 80 No 65-223 \\ Medellín, Colombia. (E-mail: mdrojas@unal.edu.co)
}

* Autor a quien se debe dirigir la correspondencia

Recibido Jul. 25, 2018; Aceptado Oct. 4, 2018; Versión final Nov. 30, 2018, Publicado Jun. 2019

\begin{abstract}
Resumen
Este artículo presenta una reflexión documentada sobre la gestión ética en las Instituciones de Educación Superior (IES) y la influencia en el establecimiento de relaciones de confianza con los diferentes grupos de interés. Las IES establecen relaciones de largo plazo con los grupos de interés, a partir de la confianza y comportamiento ético que generen las actividades misionales y colaboradores. La confianza se convierte en un activo intangible que puede ser expresado a través del capital relacional de las IES. Entre los principales resultados se encontró que el comportamiento ético y gestión de recursos institucionales favorecen la atención de los requerimientos particulares de cada uno de los grupos de interés, a partir de la integración de los valores individuales, profesionales y organizacionales. El éxito de la gestión ética en las IES se encuentra asociado con el compromiso de la alta dirección, empoderamiento de los colaboradores y gestión del riesgo.
\end{abstract}

Palabras clave: capital relacional; confianza; ética; instituciones de educación superior; tercera misión

\section{Ethics in Higher Education Institutions to Build Trusting Relationships with Stakeholders}

\begin{abstract}
This document provides a documented reflection on ethical management in Higher Education Institutions (HEI) and their influence in establishing trust relationships with different stakeholders. $\mathrm{HEl}$ establish long-term relationships with stakeholders. These relationships are generated through the trust and ethical behavior of missionary activities and partners. Trust becomes an intangible asset expressed through the relational capital of HEl. Among the main results, it was found that the ethical behavior and institutional resources management allow HEI to satisfy the particular requirements of each of the stakeholders, based on the integration of individual, professional and organizational values. The success of ethical management in HEls is associated with the commitment of directors, empowerment of employees and risk management.
\end{abstract}

Keywords: relational capital; trust; ethics; higher education institutions; third mission 


\section{INTRODUCCIÓN}

Las Instituciones de Educación Superior (IES) pueden ser vistas como organizaciones encargadas de crear, conservar y transferir conocimiento para transformar realidades económicas, políticas y sociales. Dicha transformación requiere de la identificación de los grupos de interés (stakeholders) más relevantes, necesidades del entorno y recursos organizacionales disponibles para atender estas necesidades. No obstante, la intención de suplir los requerimientos del entorno no puede comprometer el desarrollo de la razón social de la IES y el comportamiento ético de la misma. El comportamiento ético en IES depende de los valores individuales, profesionales y organizacionales. El comportamiento ético pretende evaluar cómo estos valores expresados por medio de la conducta de los individuos, tienen inferencia en el desarrollo de la sociedad (Cubillos-Vega, 2018), y en el relacionamiento con los grupos de interés. Las relaciones que se logren establecer en el largo plazo se encuentran estrechamente relacionadas con el nivel de confianza entre partes, y no de manera exclusiva entre seres humanos. Así pues, también se debe considerar la confianza que poseen los procesos y sistemas de gestión organizacional (Müller et al., 2012).

Con base en lo anterior, la confianza se convierte en el resultado de normas éticas compartidas entre partes y abarca aspectos como reciprocidad, obligación moral y compromiso hacia la comunidad (Cohen y Dienhart, 2013). Donde las normas éticas se refieren a las acciones adelantadas por la organización y que son consistentes con lo que la sociedad considera como bueno o correcto (Tseng y Chung, 2017). La confianza implica un nivel de riesgo y vulnerabilidad por parte de aquel que deposita la confianza, y un compromiso de atender los requerimientos por parte de quien la recibe. Por consiguiente, la confianza se encuentra altamente influenciada por factores sociales, culturales y políticos (Adjekum, lenca, y Vayena, 2017). Es necesario recalcar que en las IES, la confianza con los grupos de interés puede expresarse con base en el capital relacional, el cual involucra diferentes vínculos políticos, económicos y organizacionales, que se desarrollan con entidades públicas y privadas por medio de las actividades misionales de docencia, investigación y extensión (Machorro, Mercado, Cernas, y Romero, 2016; Secundo, Perez, Martinaitis, y Leitner, 2017). Se agrega que el capital relacional en las IES abarca la confianza que se genera entre los grupos de interés a partir de la imagen y reputación institucional (Machorro et al., 2016). Además, la confianza va más allá de un contrato legal que se establezca con los grupos de interés y comprende expectativas generadas entre las partes (Calvo, 2014).

Por ende, el presente artículo se basa en una reflexión documentada sobre el efecto que tiene la gestión ética de las IES en el establecimiento de relaciones de confianza con los diversos grupos de interés, teniendo en cuenta los elementos que interfieren en el capital relacional y las actividades desarrolladas en la tercera misión de las IES. La temática cobra especial relevancia al considerar la responsabilidad que poseen las IES en la proyección de un comportamiento ético que garantice la formación de ciudadanos íntegros, cubrimiento de los requerimientos sociales y articulación de grupos de interés a las actividades misionales. Para el sustento teórico de la reflexión documentada se emplearon las bases de datos Scopus, JSTOR, Redalyc y Dialnet, con base en los criterios de búsqueda capital intelectual, capital relacional, grupos de interés, tercera misión, confianza y ética, desde un punto de vista general y posteriormente específico en las IES.

\section{CAPITAL INTELECTUAL Y RELACIONAL}

El capital intelectual se constituye como factor de éxito en la era de la información. Se constituye en el elemento que permite evidenciar el conocimiento, el cual al ser gestionado genera nuevas ideas y contribuye con el logro de la estabilidad organizacional (Echeverri, Lozada, \& Arias, 2018). Además, el capital intelectual se encuentra conformado por todos aquellos activos intangibles que permiten alcanzar los objetivos organizacionales entre los cuáles se destaca el conocimiento, tecnología, relaciones, reputación, marcas y sistemas de información, los cuáles son empleados en la toma de decisiones (Isaac, Herremans, y Kline, 2010). Se han diseñado diversos modelos sobre gestión de capital intelectual. El más aceptado es el Modelo Intellectus (Ver Fig.1), el cual, en su versión más reciente considera que el capital intelectual se encuentra conformado por capital humano, capital estructural (Capital organizativo y capital tecnológico), capital relacional (Capital de negocio y capital social), capital de emprendimiento e innovación (Bueno et al., 2011).

El conocimiento es el principal activo intangible que permite generar valor y ventaja competitiva sostenida en las IES. Las IES son espacios especializados en la creación, transformación, conservación y transferencia de conocimiento. No obstante, la característica de intangibilidad permite que sólo sea posible gestionar el conocimiento por medio del capital intelectual. En cuanto a IES, la gestión de capital intelectual comprende el diseño de un grupo de indicadores claros, precisos y concisos (Leitner et al., 2014). Es de resaltar que existe una serie de indicadores que pueden extraerse del modelo Intellectus; no obstante, estos deberán adaptarse a las características propias de cada IES. Se debe agregar que el interés del presente artículo recae en el capital relacional, debido a la influencia que posee sobre los grupos de interés. El capital relacional incluye relaciones en el largo plazo con clientes, proveedores, competidores, empleados, comunidad en general y demás grupos de interés (Isaac, Herremans, y Kline, 2010). Además, el capital relacional establece 
los conocimientos que son interiorizados por la organización. El capital relacional tiene en cuenta variables como grado de conocimiento del cliente, relaciones con los empleados, grupos de interés más representativos, fidelización y satisfacción del cliente, imagen, reputación, marca y códigos de conducta organizativa (Bueno et al., 2011). De aquí que el capital relacional en IES pueda definirse como aquellos activos intangibles construidos en el relacionamiento con los diferentes grupos de interés, los cuáles se encargan de generar valor y ventaja competitiva por medio de elementos como reputación, perfil docente, direccionamiento estratégico, clasificación de grupos de interés, movilidad docente y estudiantil, convenios para el desarrollo de proyectos de investigación, productos en co-autora, reconocimientos en redes académicas, acceso a fondos de financiación y actividades de educación continua.

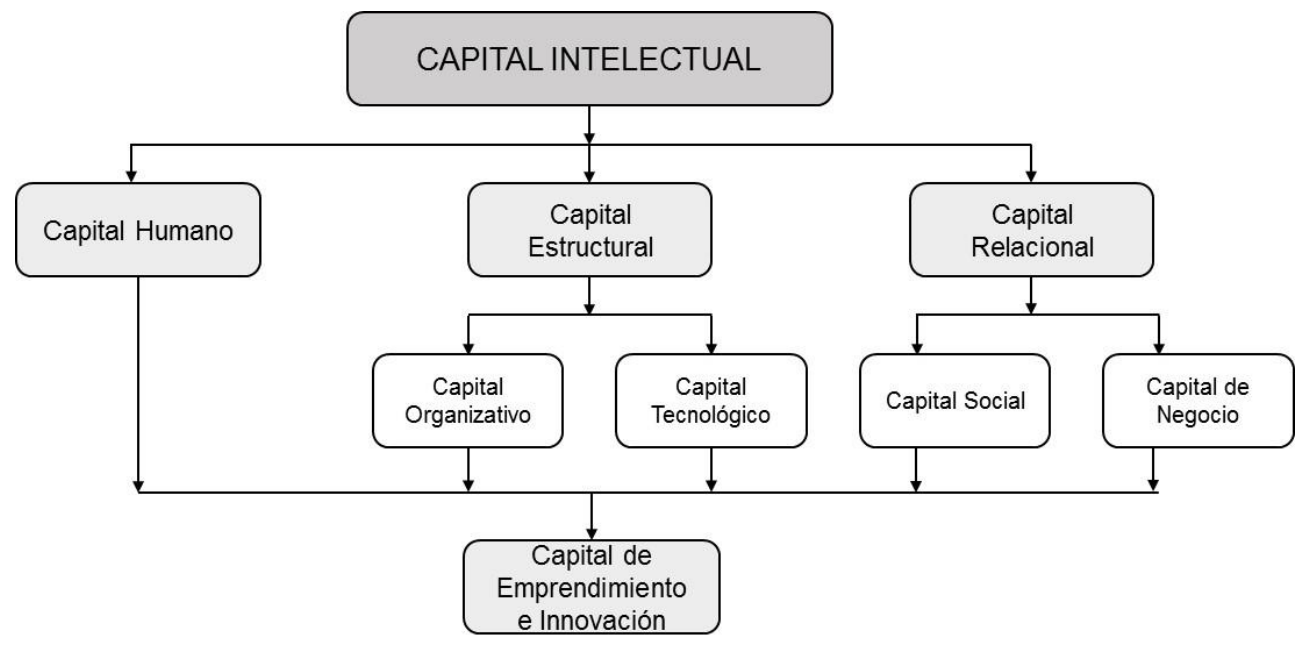

Fig. 1: Modelo Intellectus. (Adaptada de Bueno et al., 2011)

\section{GRUPOS DE INTERÉS}

Los grupos de interés son aquellos individuos o grupos que pueden afectar o son afectados por los objetivos de la organización; por ende, deben considerarse en el proceso de planeación estratégica (Freeman, 1984; Freeman, 2010). La teoría de grupo de interés contempla su identificación y la relevancia que tienen para las organizaciones (Phillips, 2003). Asimismo, la teoría de grupos de interés es inclusiva al considerar grupos primarios y secundarios que pueden amenazar o cooperar con la organización, además de considerar la actitud que se debe asumir con respecto a ellos; estos grupos se pueden dividir en cuatro categorías (Caballero, 2006; Savage, Nix, Whitehead, \& Blair, 1991): mixto, apoyo, no apoyo y marginales. Para caracterizar los grupos de interés es importante tener en cuenta tres elementos: quiénes son, qué quieren y qué están haciendo para obtenerlo, con base en las estrategias de influencia empleadas que puede ser indirecta-retención, indirecta-uso, directa-retención, directa-uso (Frooman, 1999).

En el caso de IES, los grupos de interés se encuentran conformados por la alta dirección, entidades gubernamentales, empleados, estudiantes, proveedores, competidores, patrocinadores, comunidad e intermediarios financieros. Al ser tan diversos, es importante comprender el papel individual que juegan cada uno de estos para atender los diferentes requerimientos (Marić, 2013), lo que implica la construcción de un conjunto de estrategias de relacionamiento que van más allá de la generación de convenios específicos entre las partes involucradas. Además, el desarrollo de proyectos con grupos de interés externos involucra algunos aspectos como: fomento de inversión local y propiedad, mejora de la calidad y oportunidades de educación, innovación y transferencia tecnológica, articulación de enseñanza con industria, creación de spin-off y desarrollo de fuerza laboral (Beynaghi et al., 2016). De aquí que el papel de los grupos de interés vaya más allá de la ejecución de actividades y acuerdos específicos, ya que estos grupos de interés pueden influenciar la estrategia, objetivos, calidad de la enseñanza y otras actividades institucionales (Kettunen, 2015). Por ende, cuando los requerimientos de uno de los grupos de interés cambian, se activa un proceso de retroalimentación en el cual las IES reconfiguran recursos y capacidades para llevar a cabo transformaciones organizacionales que faciliten la atención de las nuevas necesidades, convirtiéndose en un proceso de mejoramiento continuo que debe ser compartido e interiorizado por todos los colaboradores de la institución. (Ver Fig. 2.). Este proceso de mejora favorece el establecimiento de relaciones sostenibles en el largo plazo (Lee, Nguyen, Lee, Chua, y Han, 2017). Además, permite generar iniciativas de colaboración y obtención de recursos para investigación a nivel local, nacional y regional (Collins y Park, 2016).

Asimismo, el relacionamiento con los grupos de interés facilita el acceso a fondos para el desarrollo de actividades misionales por medio de donaciones, convocatorias internas, convocatorias gubernamentales y cooperación con la industria (Liefner, 2003). Es así como las IES, aplican el Modelo Triple Hélice (Etzkowitz, 2002; Etzkowitz y Leydesdorff, 2000), el cual busca estimular la competitividad de los países fortaleciendo el 
desarrollo económico basado en el conocimiento, comenzando con la restructuración organizacional de cada una de las hélices (Universidad-Empresa-Estado), pasando por la identificación de influencia de cada una en las otras, luego procede la creación de redes, para finalmente desarrollar una relación equitativa y de trabajo sinérgico entre universidad-empresa-estado. Lo anterior se obtiene a partir de un conjunto de transformaciones organizacionales, mejora continua de productos y servicios, para alcanzar una adecuada articulación entre requerimientos de la Universidad, Empresa y Estado (Ponce y Güemes, 2016). Con base en las definiciones dadas para grupos de interés por parte de (Freeman, 1984; Freeman, 2010; Kettunen, 2015), se propone la siguiente definición para grupos de interés en IES: Personas naturales y jurídicas que poseen influencia en los objetivos, desempeño y resultados organizacionales de la IES, por ende, necesitan clasificarse con base en el nivel de importancia para atender de forma oportuna y eficiente los requerimientos.

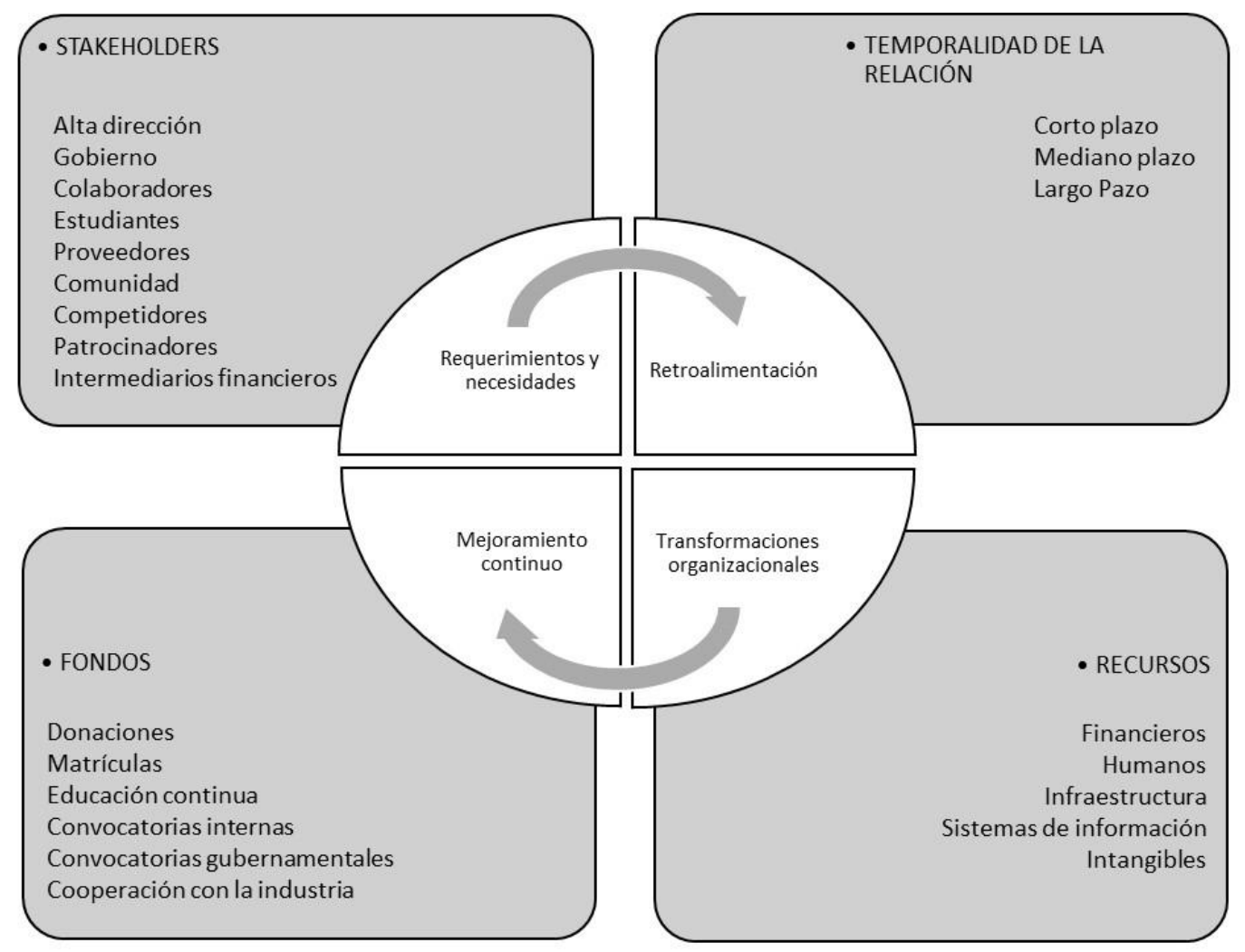

Fig. 2: Influencia grupos de interés en instituciones de Educación Superior

\section{TERCERA MISIÓN}

Es importante clasificar los grupos de interés e identificar cuáles son los relevantes para las IES. Acorde con lo anterior, la tercera misión facilita la interacción de las IES con la industria y socios regionales (Jongbloed, Enders, y Salerno, 2008). La tercera misión involucra la contribución social de IES con base en emprendimiento, transferencia tecnológica y el relacionamiento de la triple hélice (Universidad - Empresa - Estado). La tercera misión se refiere a diversas actividades no cubiertas por la primera misión, Educación, y segunda misión, Investigación, como educación continua, compromiso social, acceso público a conferencias y bienes culturales, trabajo voluntario y consultoría (Trencher, Yarime, McCormick, Doll, y Kraines, 2014). No obstante, es fundamental articular la tercera misión con la actividad misional de docencia e investigación (Jongbloed, Enders, y Salerno, 2008). La tercera misión consiste en una función de transferencia de conocimiento, así como una función de comunidad más general. En términos generales se refiere a una amplia variedad de principios y estrategias para el desarrollo económico y social (Jongbloed, Enders, y Salerno, 2008). La tercera misión en IES se encuentra conformada por tres elementos principales (Carrión, 2018): Transferencia tecnológica e innovación, educación continua y compromiso social. En este orden de ideas, se puede definir la tercera misión como el conjunto de acciones adelantadas por las IES para absorber, transferir y potenciar conocimiento institucional a los diferentes grupos de interés, de tal manera que se genere valor para las partes involucradas. La gestión de este tipo de acciones requiere volver a pensar las IES más allá de las fronteras físicas y tecnológicas, por medio del desarrollo de capacidades que les permita comprender y atender de manera adecuada las realidades del entorno sin comprometer el desarrollo de las actividades misionales.

\section{CONFIANZA Y ÉTICA ORGANIZACIONAL}

La confianza es una realidad individual, colectiva y sistémica que permite reducir complejidad y gestionar niveles de incertidumbre ante el riesgo (Luhmann, 1979). La confianza es la voluntad que tiene una parte de 
ser vulnerable a las acciones de otra parte con base en las expectativas de que la otra parte llevará a cabo una acción particular sin necesidad de ser monitoreada o controlada, se encuentra conformada por habilidad, benevolencia e integridad (Mayer, Davis \& Schoorman, 1995). La confianza es el compromiso que adquiere una parte de colaborar y cumplir las obligaciones pactadas en las relaciones, la cuales se pueden categorizar en notoriedad, exploración, expansión, compromiso y disolución (Dwyer, Schurr \& Oh, 1987). La confianza es está conformada por credibilidad (Confianza en la palabra de los socios) y benevolencia (Interés en el bienestar común) (Doney \& Cannon, 1997). La confianza es vista como un bien intangible de la organización que al articularse con la responsabilidad social facilita la toma de decisiones en las organizaciones (Calvo, 2014). Además, la confianza puede ser descrita a partir de una relación tripartita dónde se destaca quien deposita la confianza, quien recibe la confianza y la ejecución de una tarea específica (Adjekum et al., 2017). Así pues, la confianza se refiere a la percepción de la seguridad que tienen los colaboradores con respecto a si la organización o los colegas serán confiables, afectando el desempeño organizacional y la intención del colaborador de permanecer en la organización (Tseng y Chung, 2017).

La confianza se emplea para describir al menos cinco tipos diferentes de relaciones (Müller et al., 2012): individuos, grupos, entidades sociales, relaciones entre individuos; y relaciones entre individuos y grupos. Por ende, la confianza expresa la voluntad de una parte, de ser vulnerable a las acciones de otra parte, basada en expectativas de que la otra realizará una acción particular (Cohen y Dienhart, 2013; Mayer, Davis, y Schoorman, 1995). La confianza es un fenómeno moral cuando A confía en B para realizar algo, A invita a B a reconocer y aceptar una obligación, cuando $B$ acepta la invitación, $B$ toma la obligación y la confianza se convierte en obligación (Cohen y Dienhart, 2013).La confianza entre individuos u organizaciones se encuentra ligada a un conjunto de riesgos asociados a la materialización de las expectativas generadas entre quien deposita la confianza y quien la recibe (Adjekum et al., 2017; Cohen y Dienhart, 2013). Los riesgos potenciales se asumen de manera voluntaria por cada una de las partes interesadas. De aquí que el adecuado conocimiento de los grupos de interés ayude a disminuir la aversión al riesgo en las diferentes actividades de relacionamiento. El nivel de confianza entre los grupos de interés permite que estos asuman mayores riesgos al momento de cooperar. De esa forma, identificar y fortalecer esta clase de recursos intangibles facilita la reducción de costos de transacción, optimizar el desempeño de la estrategia organizacional a mediano y largo plazo, alcanzar los objetivos organizacionales, acercándose así a la consecución del beneficio óptimo (Calvo, 2014). Además, se han diseñado diversos modelos para medir e implementar sistemas de ética y confianza en las organizaciones (Ver Tabla 1.). Es importante considerar que los modelos deben adaptarse a las particularidades de cada organización, incluyendo las IES.

Tabla 1: Modelos de ética y confianza

\begin{tabular}{|c|c|c|c|}
\hline \multicolumn{2}{|c|}{ Dimensión } & \multirow[b]{2}{*}{ Componentes } & \multirow[b]{2}{*}{ Autor } \\
\hline Ética & $\begin{array}{l}\text { Con- } \\
\text { fianza }\end{array}$ & & \\
\hline$x$ & $X$ & $\begin{array}{l}\text { Confianza en la alta dirección. } \\
\text { Intención ética / no ética. } \\
\text { Estrategia orientada al cliente. }\end{array}$ & $\begin{array}{l}\text { Schwepker y } \\
\text { Schultz, } 2013\end{array}$ \\
\hline \multirow[t]{3}{*}{$x$} & $x$ & $\begin{array}{l}\text { Liderazgo: Transformacional, transaccional. } \\
\text { Confianza organizacional: basada en el conocimiento, identidad y cálculo. } \\
\text { Comportamiento ético / no ético: Menor o mayor nivel. }\end{array}$ & Trong, 2012 \\
\hline & $x$ & $\begin{array}{l}\text { Variables de estatus: Historia de las relaciones, distancia entre firmas. } \\
\text { Variables de proceso: Autonomía, multiculturalismo, velocidad de integración, } \\
\text { beneficios percibidos, calidad de la comunicación. } \\
\text { Integridad organizacional: Habilidad, benevolencia, apertura, congruencia de } \\
\text { valores. } \\
\text { Salidas de integración sociocultural: Compromiso, satisfacción, aceptación del } \\
\text { cambio, intención de permanecer, cooperación, transferencia de información, } \\
\text { desempeño laboral. } \\
\text { Desempeño después de la adquisición: Crecimiento de las ventas, utilidades } \\
\text { esperadas. }\end{array}$ & $\begin{array}{l}\text { Stahl, } \\
\text { Larsson, } \\
\text { Kremershof, y } \\
\text { Sitkin, } 2011\end{array}$ \\
\hline & $x$ & $\begin{array}{l}\text { Integridad organizacional: Competencia, buena voluntad, integridad, justicia y } \\
\text { respeto. } \\
\text { Tendencia a la confianza por parte de los colaboradores. }\end{array}$ & $\begin{array}{l}\text { McLeary y } \\
\text { Cruise, } 2015\end{array}$ \\
\hline$x$ & & $\begin{array}{l}\text { Principios de clima ético: Individual, legal y cosmopolita. } \\
\text { Individualismo, relativismo. } \\
\text { Compromiso: Afectivo, normativo y de continuidad. } \\
\text { Egoísmo individual, egoísmo cosmopolita y benevolencia. }\end{array}$ & $\begin{array}{l}\text { Putranta y } \\
\text { Kingshott, } \\
2011\end{array}$ \\
\hline $\mathrm{X}$ & & $\begin{array}{l}\text { Juicio ético: Ética organizacional, ética del colaborador, atención moral reflexiva. } \\
\text { Intención ética. }\end{array}$ & $\begin{array}{l}\text { Culiberg y } \\
\text { Mihelič, } 2016\end{array}$ \\
\hline
\end{tabular}


En lo referente a la ética empresarial, esta es el reflejo ético de un negocio con respecto al comportamiento organizacional y los impactos en la sociedad, teniendo en cuenta valores corporativos sobre integridad, responsabilidad, honestidad, confianza, equidad, cooperación, mutualidad, profesionalismo y comunicación abierta (Su, 2014). La ética contribuye a que los colaboradores de una organización identifiquen que las acciones poseen consecuencias en ellos y en el entorno (Osorio \& Rodríguez, 2018). Los valores éticos, como la privacidad, la transparencia y la equidad, son probablemente factores determinantes de la confianza tanto a nivel individual como colectivo (Adjekum et al., 2017). Asimismo, la ética empresarial se refiere al comportamiento responsable de las organizaciones en todos los entornos en los cuales se desenvuelve; es decir, que todos los colaboradores de la organización deben compartir compromisos mutuos, afectos, valores, lealtad, transparencia, sentido de pertenencia, cuidado y responsabilidad hacia los demás, ocupándose de los aspectos de naturaleza moral existentes en los negocios (Zapata, 2015). De aquí que, entre los elementos que componen la ética empresarial se encuentren aspectos relacionados con el clima y la gestión de la organización, como estilo de dirección y toma de decisiones, gestión del talento humano, interlocución y comunicación; y estructura organizacional (Cubillos-Vega, 2018). Asimismo, la ética organizacional y la inclusión en las actividades diarias de los colaboradores, puede reflejarse por medio de los códigos de ética, los cuales fallan con regularidad, resultando más exitosa la autorregulación y construcción de una cultura de confianza (Müller et al., 2012). Sin embargo, las reglas éticas en el trabajo mejoran la confianza de los colaboradores, al tener gerentes más responsables y confiables (Tseng y Chung, 2017). Con base en lo anterior, la ética organizacional posee un impacto positivo en el capital intelectual, ya que permite el desarrollo de recursos intangibles asociados al conocimiento (Su, 2014). Las organizaciones con valores y conductas éticas construyen confianza con los diferentes grupos de interés, lo que facilita mejores interacciones.

De forma particular, la conducta ética es esencial para la investigación científica en IES ya que permite fomentar colaboración, cooperación y confianza entre investigadores y sociedad (Shamoo \& Resnik, 2009). No obstante, la presión para incrementar la productividad académica de los investigadores puede contribuir con alteraciones en el comportamiento ético, como plagio y fraude (Tijdink, Verbeke \& Smulders, 2014). Además, el incremento en los requerimientos para generar productos en co-autoría puede generar otras alteraciones a la ética en IES como: no darle crédito en un trabajo académico a una persona que si lo merece, darle crédito académico a un individuo que no lo merece; y conflictos basados en el género (Youtie \& Bozeman, 2014). Por otra parte, la ética en IES contempla calidad de enseñanza y profesionalismo de docentes y demás colaboradores (Kafi, Motallebzadeh \& Ashraf, 2018).

En consecuencia, el comportamiento ético en las IES es el resultado de los valores compartidos entre los colaboradores y el compromiso de los directivos. Además, el comportamiento ético de los colaboradores y directivos no es estático, varía conforme a la incertidumbre del entorno y requerimientos de los grupos de interés. Se expresa por medio de diferentes niveles de empoderamiento que dependerán de la normatividad y mecanismos de autorregulación de los individuos. De forma similar, el comportamiento ético en las IES genera activos intangibles relacionados con el conocimiento entre los cuales se destaca el capital relacional, el cual se deriva del establecimiento de relaciones de confianza con los diferentes grupos de interés. De aquí que el acceso ha dicho relacionamiento se convierta en un proceso iterativo, caracterizado por la expectativa, riesgo y responsabilidades de cada una de las partes involucradas. Más aun, el comportamiento ético reflejado en las acciones adelantadas por cada uno de los colaboradores, se convierte en el driver central para que las IES construyan relaciones en el largo plazo con los grupos de interés, las cuales contribuyen con el desarrollo de las actividades misionales de docencia, investigación y extensión. Es importante que las IES incorporen la gestión ética a la toma de decisiones y estrategia institucional, donde se identifiquen los recursos tangibles e intangibles que garantizarán la atención oportuna de los requerimientos de los grupos de interés, sin comprometer el desarrollo de las actividades diarias de la IES. De forma especial, las IES deben diseñar mecanismos que permitan alinear la conducta de los colaboradores con el comportamiento ético institucional.

\section{CONCLUSIONES}

El establecimiento de relaciones de confianza entre Instituciones de Educación Superior (IES) y los diferentes grupos de interés, implica identificación de los requerimientos particulares de cada uno de los grupos de interés y reconocimiento del comportamiento ético de todos los integrantes de la comunidad académica. El nivel de confianza se expresa como el resultado de la integración de los valores individuales, profesionales y organizacionales. A su vez, involucra la renovación de la estructura y gestión institucional, a fin de dar una adecuada gestión a los recursos físicos, humanos, financieros y activos intangibles. La implementación de un sistema ético o de confianza organizacional, requiere del compromiso de la alta dirección y líderes de proceso; además, del empoderamiento que se brinde a los demás colaboradores de la IES. Es de considerar que el éxito de estos sistemas dependerá de las características individuales de los colaboradores y el nivel de identidad que adquieran con respecto a los lineamientos éticos o de confianza institucionales. Más aun, el comportamiento ético es dinámico y genera valor en las IES; por ende, se debe incluir en la gestión estratégica institucional, de forma especial en aquellos elementos vinculados con los activos que se generan en el relacionamiento con los diferentes grupos de interés. 
La tercera misión de las IES se convierte en el mecanismo de articulación entre la satisfacción de las necesidades de los grupos de interés más relevantes y la transferencia de conocimiento por medio de diferentes procesos como educación continua, compromiso social, transferencia tecnológica y acceso a fondos para la ejecución de diferentes actividades misionales. La implementación de este mecanismo de articulación involucra una serie de riesgos para la parte que deposita confianza y para aquella quien la recibe. De aquí que el conocimiento mutuo se convierta en una alternativa para disminuir los riesgos asociados al proceso de relacionamiento. Se identificaron algunas limitaciones en el desarrollo de la reflexión documentada, entre las cuales se destaca la escasez de información sobre investigaciones teóricas y empíricas que articulen las variables de confianza, ética y gestión de capital relacional en IES. Además, el nivel de madurez de gestión de activos intangibles y características particulares de cada IES, dificulta la generalización de los resultados de investigación. En este orden de ideas, se propone como trabajo futuro el diseño de un modelo conceptual y aplicación de la metodología Partial Least Square (PLS), a fin de analizar al relación existente entre gestión ética, tercera misión y toma de decisiones que favorezcan el establecimiento de relaciones de confianza en el largo plazo con los diferentes grupos de interés en las IES.

\section{REFERENCIAS}

Adjekum, A., M. lenca y E. Vayena, What Is Trust? Ethics and Risk Governance in Precision Medicine and Predictive Analytics, doi:10.1089/omi.2017.0156, OMICS: A Journal of Integrative Biology, 21(12), 704-710 (2017)

Beynaghi, A., G. Trencher, F. Moztarzadeh, M. Mozafari, R. Maknoon y W. Leal Filho, Future sustainability scenarios for universities: moving beyond the United Nations Decade of Education for Sustainable Development, doi: 10.1016/j.jclepro.2015.10.11, Journal of Cleaner Production, 112, 3464-3478 (2016)

Bueno, E., C. Murcia y otros cinco autores, Modelo Intellectus: Medición y Gestión del Capital Intelectual, IADE, Madrid, España (2011)

Caballero, G., Análisis de los stakeholders: una reflexión sobre el poder de influencia en la organización, Revista Icade, Revista de las Facultades de Derecho y Ciencias Económicas y Empresariales, 0(68), 101-122 (2006)

Calvo, P., Bussiness ethics, social responsibility and Communication Goods, Tópicos, México, (47), 199-232 (2014)

Carrión, A., Una Universidad Socialmente Responsable, En Responsabilidad social corporativa: una mirada integral en América Latina, 11 - 19, Cali, Colombia (2018)

Cohen, M.A. y J. Dienhart, Moral and Amoral Conceptions of Trust, with an Application in Organizational Ethics, doi: 10.1007/s10551-012-1218-5, Journal of Business Ethics, 112(1), 1-13 (2013)

Collins, F.L. y G.-S. Park, Ranking and the multiplication of reputation: reflections from the frontier of globalizing higher education, doi: 10.1007/s10734-015-9941-3, Higher Education, 72(1), 115-129 (2016)

Cubillos-Vega, C., Ética organizacional aplicada. Estudio de caso de una organización de servicios sociales en España, doi: 10.15446/innovar.v28n67.68616, Innovar, 28(67), 111-121 (2018)

Culiberg, B. y K. K. Mihelič, Three ethical frames of reference: insights into Millennials' ethical judgements and intentions in the workplace, doi: 10.1111/beer.12106, Business Ethics: A European Review, 25(1), 94-111 (2016)

Doney, P.M. y J. P. Cannon, An Examination of the Nature of Trust in Buyer-Seller Relationships, doi: 10.2307/1251829, Journal of Marketing, 61(2), 35-51 (1997)

Dwyer, F.R., P.H. Schurr y S. Oh, Developing Buyer-Seller Relationships, doi: 10.2307/1251126, J. of Marketing, 51(2), 11-27 (1987)

Echeverri, A., N. Lozada y J.E. Arias, Incidencia de las Prácticas de Gestión del Conocimiento sobre la Creatividad Organizacional, doi: 10.4067/S0718-07642018000100059, Información Tecnológica, 29(1), 71-82 (2018)

Etzkowitz, H., The Triple Helix of University - Industry - Government, Implications for Policy and Evaluation, Institutet för studier av utbildning och forskning (2002)

Etzkowitz, H. y L. Leydesdorff, The dynamics of innovation: from National Systems and "Mode 2" to a Triple Helix of university-industry-government relations, doi: 10.1016/S0048-7333(99)00055-4, Research Policy, 29(2), 109-123 (2000)

Freeman, R.E., Strategic Management: A Stakeholder Approach, Boston, Pitman (1984)

Freeman, R.E., Strategic Management: A Stakeholder Approach, Cambridge University Press (2010)

Frooman, J., Stakeholder Influence Strategies, doi:10.2307/259074, The Academy of Management Review, 24(2), 191-205 (1999)

Isaac, R.G., I.M. Herremans y T.J. Kline, Intellectual Capital Management Enablers: A Structural Equation Modeling Analysis, doi: 10.1007/s10551-009-0227-5, Journal of Business Ethics, 93(3), 373-391 (2010)

Jongbloed, B., J. Enders y C. Salerno, Higher education and its communities: Interconnections, interdependencies and a research agenda, doi:10.1007/s10734-008-9128-2, Higher Education, 56(3), 303-324 (2008)

Kafi, Z., K. Motallebzadeh y H. Ashraf, University Instructors' Teaching Experience and Their Perception of Professional Ethics: Postulating a Model, doi: 10.12973/iji.2018.11417a, International Journal of Instruction, 11(4), 257-270 (2018) 
Kettunen, J., Stakeholder Relationships in Higher Education, doi: 10.1080/13583883.2014.997277, Tertiary Education and Management, 21(1), 56-65 (2015)

Lee, S., H.N. Nguyen, K.-S. Lee, B.-L. Chua y H. Han, Price, people, location, culture and reputation: determinants of Malaysia as study destination by international hospitality and tourism undergraduates, doi: 10.1080/14766825.2017.1336242, Journal of Tourism and Cultural Change, 1-13 (2017)

Leitner, K., S.E. Perez y otros seis autores, A strategic approach for intellectual capital management in European universities, Executive Agency for Higher Education, Research, Development and Innovation Funding, Bucarest, Rumania (2014)

Liefner, I., Funding, resource allocation and performance in higher education systems, doi: 10.1023/A:1027381906977, Higher Education, 46(4), 469-489 (2003)

Luhmann, N., Trust and Power: Two Works, Wiley (1979)

Machorro, F., P. Mercado, D. A .Cernas y M. V. Romero, Influencia del capital relacional en el desempeño organizacional de las instituciones de educación superior tecnológica, doi: 10.15446/innovar.v26n60.55531, Innovar, 26(60), 35-50 (2016)

Mayer, R.C., J.H. Davis y F. D. Schoorman, An Integrative Model of Organizational Trust, doi: 10.2307/258792, The Academy of Management Review, 20(3), 709-734 (1995)

Marić, I., Stakeholder Analisys of Higher Education Institutions. Interdisciplinary Description of Complex Systems, doi: 10.7906, INDECS, 11(2), 217-226 (2013)

Mayer, R.C., J.H. Davis y F.D. Schoorman, An Integrative Model of Organizational Trust, doi: 10.2307/258792, The Academy of Management Review, 20(3), 709-734 (1995)

McLeary, C.N. y P.A. Cruise, A context-specific model of organizational trust: An examination of cognitive and socioaffective trust determinants in unique cultural settings, doi: 10.1108/CCM-11-2013-0180, Cross Cultural Management: An International Journal, 22(2), 297-320 (2015)

Müller, R., E. S. Andersen, Ø. Kvalnes, J. Shao, S. Sankaran y J.R. Turner, The Interrelationship of Governance, Trust, and Ethics in Temporary Organizations, doi: 10.1002/pmj.21350, Project Management Journal, 44(4), 26-44 (2012)

Osorio, V. y M. del P. Rodríguez, Modelo para la Evaluación de Programas de Ética Organizacional en Universidades, doi:10.4067/S0718-07642018000100059, Información Tecnológica, 29(1), 59-70 (2018)

Phillips, R., Stakeholder Theory and Organizational Ethics, Berrett-Koehler Publishers (2003)

Ponce, I. y D. Güemes, Identification of key factors of academia in the process of linking in the triple helix of innovation model in Mexico, a state of the art matrix, Nova Scientia, 8(16), 246-277 (2016)

Putranta, M.P. y R.P.J. Kingshott, The Relationships Between Ethical Climates, Ethical Ideologies and Organisational Commitment Within Indonesian Higher Education Institutions, doi: 10.1007/s10805-010-9122-Z, Journal of Academic Ethics, 9(1), 43-60 (2011)

Savage, G.T., T. W. Nix, C. J. Whitehead y J. D. Blair, Strategies for Assessing and Managing Organizational Stakeholders, The Executive, 5(2), 61-75 (1991)

Schwepker, C.H. y R.J. Schultz, The impact of trust in manager on unethical intention and customer-oriented selling, doi: 10.1108/08858621311313938, Journal of Business \& Industrial Marketing, 28(4), 347-356 (2013)

Secundo, G., S. Perez, Ž. Martinaitis y K. Leitner, An Intellectual Capital framework to measure universities' third mission activities, doi: 10.1016/j.techfore.2016.12.013, Technological Forecasting and Social Change, 123, 311 - 320 (2017)

Shamoo, A.E. y D.B. Resnik, Scientific Research and Ethics, Oxford University Press (2009)

Stahl, G.K., R. Larsson, I. Kremershof y S.B. Sitkin, Trust dynamics in acquisitions: A case survey, doi: 10.1002/hrm.20448, Human Resource Management, 50(5), 575-603 (2011)

Su, H.-Y., Business Ethics and the Development of Intellectual Capital, doi: 10.1007/s10551-013-1623-4, Journal of Business Ethics, 119(1), 87-98 (2014)

Tijdink, J.K., R. Verbeke e Y.M. Smulders, Publication Pressure and Scientific Misconduct in Medical Scientists, doi: 10.1177/1556264614552421, Journal of Empirical Research on Human Research Ethics, 9(5), 64-71 (2014)

Trencher, G., M. Yarime, K.B. McCormick, C.N.H. Doll y S.B. Kraines, Beyond the third mission: Exploring the emerging university function of co-creation for sustainability, doi: 10.1093/scipol/sct044, Science and Public Policy, 41(2), 151$179(2014)$

Trong, L., The linkages among leadership, trust, and business ethics, doi: 10.1108/17471111211196629, Social Responsibility Journal, 8(1), 133-148 (2012)

Tseng, L.-M. y C.-E. Chung, The role of explicit ethics institutionalization and management accountability in influencing newcomer trust, doi:10.1108/IJOA-09-2016-1069, International Journal of Organizational Analysis, 25(4), 613-627 (2017)

Youtie, J. y B. Bozeman, Social dynamics of research collaboration: norms, practices, and ethical issues in determining co-authorship rights, doi: 10.1007/s11192-014-1391-7, Scientometrics, 101(2), 953-962 (2014)

Zapata, A. D., Reflexiones Desde la Ética y la Responsabilidad Social Empresarial, Advocatus, 12(25), 195-20 (2015) 